\title{
Driving Adsorbed Gold Nanoparticle Assembly by Merging Lipid Gel/Fluid Interfaces
}

\author{
Feng Wang, ${ }^{1}$ Dennis E. Curry ${ }^{2}$ and Juewen Liu ${ }^{1 *}$ \\ Departments of Chemistry ${ }^{1}$ and Biology ${ }^{2}$, Waterloo Institute for Nanotechnology, Waterloo, Ontario, Canada, N2L \\ $3 \mathrm{G} 1$.
}

Supporting Information Placeholder

\begin{abstract}
Surface forces between inorganic nanoparticles and lipid bilayer is of great relevance to biophysics, medicine, and nanobiotechnology. Adsorbed nanoparticles may influence the fluidity of the underlying lipids, which may in turn influence nanoparticle assembly. Herein three types of lipids $\left(\mathrm{DOPC}, T_{\mathrm{c}}=-20^{\circ} \mathrm{C}\right.$; DMPC, $T_{\mathrm{c}}=23^{\circ} \mathrm{C}$, and DPPC, $T_{\mathrm{c}}=$ $41{ }^{\circ} \mathrm{C}$ ) are used, all with the same phosphocholine (PC) headgroup. Gold nanoparticle (AuNP) color change is monitored as a function of lipid phase transition temperature $\left(T_{\mathrm{c}}\right)$, surface ligands on AuNPs, and temperature. Liposomes with higher fluidity induce much faster aggregation of AuNPs. Aside from the kinetic aspect of faster diffusion on fluid bilayers, this faster color change is attributed to the local lipid gelation and merging of gelled regions to eliminate the interface between different lipid phases.
\end{abstract}

\section{Introduction}

Lipid bilayers represent a unique type of surface, since individual lipid molecules diffuse quickly within the bilayer membrane.1, 2 Such lateral diffusion is not easily achieved by other surfaces (e.g. inorganic materials). Lipid diffusion has extremely important biological implications for membrane protein assembly and signaling. Molecules covalently attached to a lipid diffuse together with the lipid. ${ }^{-5}$ An interesting situation arises if a molecule is not covalently attached, but physisorbed by the bilayer. The intermolecular force might affect the diffusion of the molecule on the bilayer surface. However, it is quite difficult to adjust the adsorption interaction strength of single molecules. ${ }^{6,7}$

Recent research on the adsorption of inorganic NPs by lipid membranes may provide a platform to study this problem. For example, zwitterionic phosphocholine (PC) liposomes can adsorb a diverse range of nanomaterials, including latex beads, ${ }^{8,} 9 \mathrm{TiO}_{2},{ }^{10-12}$ graphene oxide, ${ }^{13}, 14$ nano-diamonds, ${ }^{15}$ and gold NPs (AuNPs). ${ }^{16-22}$ Among these materials, AuNPs are particularly interesting: 1) the surface chemistry of AuNPs can be readily controlled by capping with thiol ligands; 2) AuNPs display distance-dependent color due to surface plasmon coupling, allowing convenient visual monitoring;23 and 3) AuNPs have strong van der Waals interaction (i.e. large Hamaker constant) with other surfaces thus making strong physisorption possible. ${ }^{24}$ In this work, we focus on the interesting case of non-tethered AuNPs, and the kinetic and thermodynamic aspect of lipid fluidity.

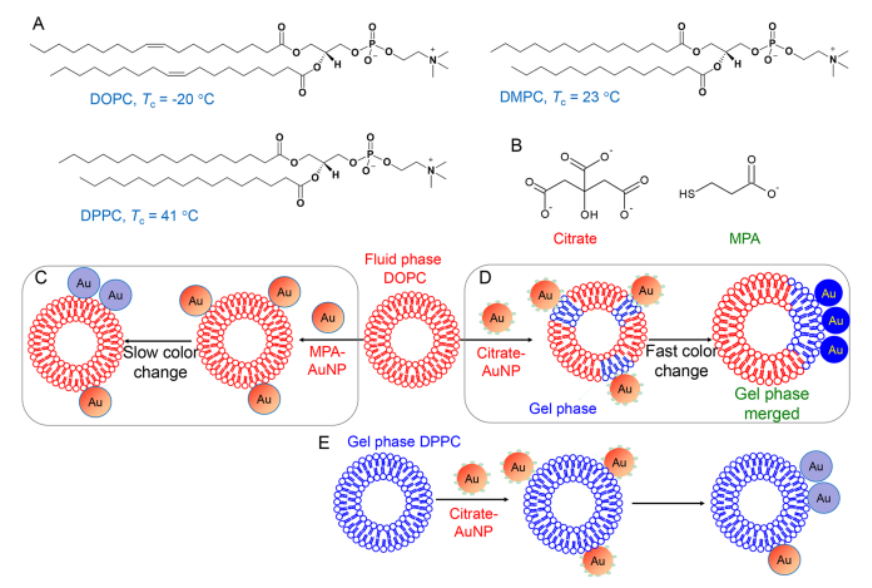

Figure 1. (A) The structure of the three types of PC lipids used in this work with different $T_{\mathrm{c}}$ 's. (B) The structure of citrate and MPA for capping AuNPs. Models of (C) MPAAuNP adsorption by DOPC; (D) citrate-AuNP adsorption by DOPC, inducing local gelation and emerging of gelled areas; and (E) citrate-AuNP adsorbed by DPPC. The gel phased lipids are in blue and the fluid phase in red.

\section{Results and Discussion}

To study the effect of lipid diffusion, a critical parameter is the phase transition temperature $\left(T_{\mathrm{c}}\right)$. A lipid membrane displays two main phases. Above $T_{\mathrm{c}}$, lipids are in the fluid phase, and can diffuse quickly within the bilayer. Below $T_{\mathrm{c}}$, lipids are in the gel phase, and diffuse more slowly. In this study, we employed three types of lipids (DOPC, $T_{\mathrm{c}}=-20{ }^{\circ} \mathrm{C}$; DMPC, $T_{\mathrm{c}}=23{ }^{\circ} \mathrm{C}$, and DPPC, $\left.T_{\mathrm{c}}=41^{\circ} \mathrm{C}\right)$, all with the same PC headgroup (Figure $1 \mathrm{~A}$ ). Using these lipids, $\sim 100 \mathrm{~nm}$ diameter liposomes were prepared by extrusion (Figure $\mathrm{S}_{1}$ ).

To have an initial understanding, each liposome was respectively mixed with citrate-capped $13 \mathrm{~nm}$ AuNPs (AuNP: liposome = 10:1). After $2 \mathrm{~min}$, the color of the AuNPs changed from red to blue with DOPC, and to purple with DMPC (Figure $2 \mathrm{~A}$ ), indicating extensively AuNP aggregation. The surface charge of these PC liposomes are close to zero (Figure S2), allowing quick AuNP adsorption, which is supported by the fast color change. Interestingly, little color change was observed with DPPC. The main difference between these liposomes is lipid fluidity. We used a low concentration of salt ( $2 \mathrm{mM})$ to avoid AuNP aggregation in solution. Indeed, the control sample without liposome remained red. There- 
fore, aggregation was not directly induced by salt, but promoted by the liposomes.

To have a quantitative understanding, the kinetics of AuNP color change was followed by UV-vis spectroscopy. Free citrate-capped AuNPs have a characteristic surface plasmon peak at $520 \mathrm{~nm}$ (Figure $2 \mathrm{~B}$, black spectrum). Upon addition of fluid DOPC liposomes, a new peak at $600 \mathrm{~nm}$ quickly emerged, while the intensity of this $520 \mathrm{~nm}$ peak barely charged. The increased extinction at longer wavelengths indicates AuNP aggregation. We plotted the kinetics of the growth of this $600 \mathrm{~nm}$ peak (Figure $2 \mathrm{C}$, black trace); most of the color change occurred in the first $2 \mathrm{~min}$. On the other hand, the shift was very moderate in the presence of the gel phase DPPC, leaving DMPC in between. This kinetic trend is consistent with the visual color change.

To further confirm the effect of lipid fluidity, we tested the DMPC liposomes at three temperatures (Figure 2D). Faster color change was observed at higher temperature. Since the lipid composition is the same and the only difference is temperature, this further supports the importance of lipid fluidity. To quantitatively measure the fluidity effect, we also incubated the DMPC/AuNP mixture for 15 min at various temperatures before recording their spectra (Figure $2 \mathrm{E}$ ). The color change was initially small at low temperature (e.g. below $15{ }^{\circ} \mathrm{C}$ ). Between 15 and $20{ }^{\circ} \mathrm{C}$, the color change has a strong temperature dependency, while saturated color change occurred at above $20{ }^{\circ} \mathrm{C}$, which is slightly below the $T_{\mathrm{c}}$ of DMPC $\left(23^{\circ} \mathrm{C}\right)$. This slight difference might be attributed to the temperature fluctuation in our waterbath during the 15 min incubation. Since AuNP aggregation is irreversible, we were measuring the highest temperature reached at the specified average temperature. Another possibility is that we may be probing the presence of the fluid phase by the AuNP color change, and the fluid phase emerges below the $T_{\mathrm{c}}$.
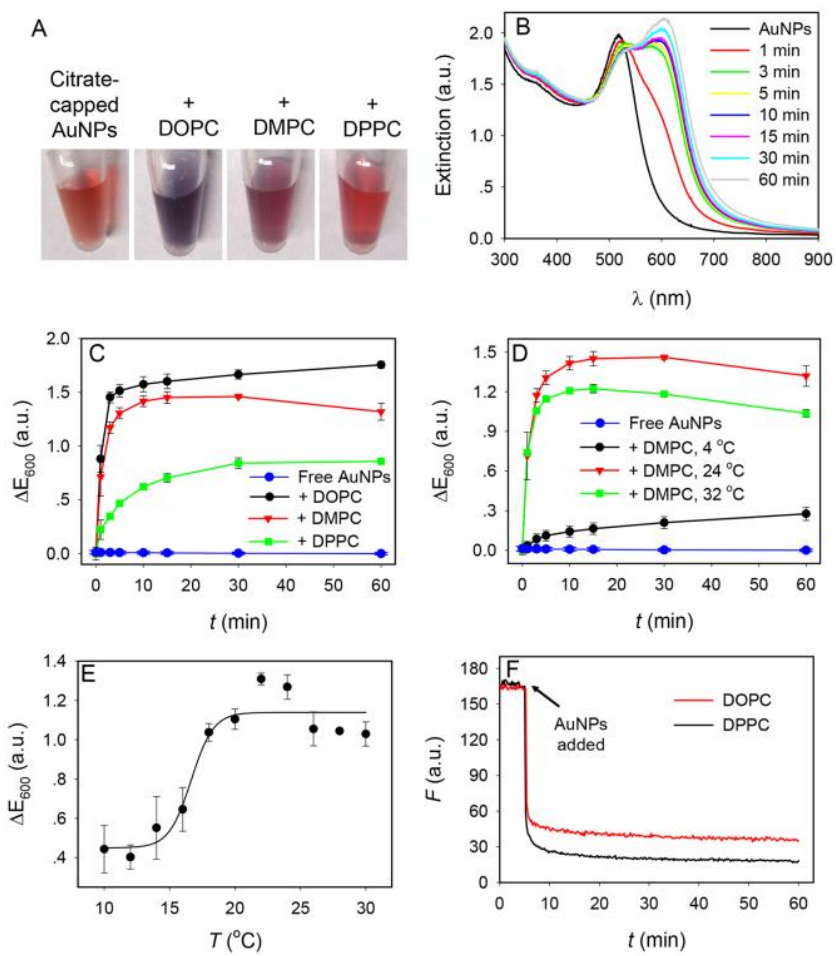

Figure 2. (A) The color of citrate-AuNPs when mixed with various liposomes $\left(\mathrm{AuNP}=5 \mathrm{nM}\right.$; liposome $=0.5 \mathrm{nM} ; \mathrm{Na}^{+}=2$
$\mathrm{mM}$ ). Blue color indicates aggregated AuNPs. (B) UV-vis spectra of DOPC mixed with citrate-capped AuNPs. Kinetics of $600 \mathrm{~nm}$ extinction increase after mixing citrate-AuNPs with $(C)$ various liposomes at room temperature, or (D) with DMPC liposomes at various temperatures. Some of the lines curve down at later time points due to precipitation of AuNPs after their accumulation on liposomes. (E) $600 \mathrm{~nm}$ extinction change after mixing AuNPs with DMPC at various temperatures for $15 \mathrm{~min}$. (F) Fluorescence of NBD-labeled liposomes (o.5 nM) after adding citrate-AuNPs (o.1 nM).

To test whether the observed color change difference was due to the initial AuNP adsorption kinetics, we prepared NBD-labeled DOPC and DPPC liposomes. Since the NBD is labeled on the lipid tail, the artifact of AuNP adsorption by the dye is avoided. AuNP adsorption is accompanied with fluorescence quenching (Figure $2 \mathrm{~F}$ ); adsorption is instantaneous for both liposomes and most fluorescence quenching occurred in the first minute. Since AuNPs have a strong absorption peak at $520 \mathrm{~nm}$, overlapping with the NBD emission, the fluorescence drop might be due to this inner filter effect. To further test this, we also used a centrifugation method to precipitate AuNPs but not free liposomes. (Figure $\mathrm{S}_{3}$ ). In this experiment, both liposomes were quickly cleared from the supernatant. Therefore, PC liposomes adsorb AuNPs very quickly, regardless of lipid fluidity.

Next, we tested the effect of AuNP surface chemistry, which allows modulation of adsorption affinity. We capped AuNPs with MPA (Figure 1B). MPA positions AuNPs slightly away from the lipid surface, reducing the van der Waals force. ${ }^{21}$ It needs to be noted that the citrate molecules on the AuNPs can be easily displaced, allowing AuNPs to approach the liposome surface more closely with a larger van der Waals force. ${ }^{21}$

When MPA-capped AuNPs were mixed with DOPC liposomes, the extent of color change was indeed much less (Figure $3 \mathrm{~A}$ ). MPA-AuNPs can also be quickly adsorbed by PC liposomes as indicated by the fluorescence quenching experiment (Figure $\mathrm{S}_{4}$ ). As a further control, we also measured salt-induced aggregation of citrate-AuNP and MPA-AuNPs in the absence of any liposomes (Figure ${ }_{3} \mathrm{~B}$, Figure $\mathrm{S}_{5}$ ). Both types of AuNPs have a similar tolerance for salt and color change starts at $\sim 25 \mathrm{mM} \mathrm{NaCl}$. Therefore, the slower aggregation of MPA-AuNPs cannot be attributed to its higher colloidal stability, but due to its decreased interaction strength with the liposome surface.

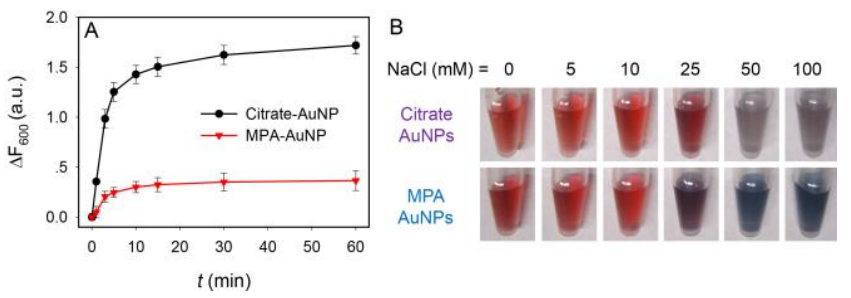

Figure 3. (A) Kinetics of color change of citrate and MPAcapped AuNPs after mixing with DOPC liposomes. (B) Photographs of citrate and MPA-capped AuNP aggregation by salt.

To further understand the assembly of AuNPs on liposome, we carried out transmission electron microscopy 
(TEM) studies. Figure 4A shows a cryo-TEM image of citratecapped AuNPs mixed with DOPC liposomes, and the AuNPs formed large clusters explaining the color change. This also indicates that AuNPs are aggregated on the liposomes instead of in solution. Since cryo-TEM is a slow and resourcedemanding technique, we also carried out normal TEM. Figure $4 \mathrm{~B}$ shows the same sample and we also observe a similar type of AuNP clustering, but then the liposomes were invisible. On the other hand, MPA-AuNPs are more dispersed on DOPC, showing only small clusters of a few AuNPs or short chains without forming large compact 2-D aggregates (Figure $\left.{ }_{4} C\right)$. This is consistent with their difference in UV-vis spectra. Similarly, citrate-AuNPs remain quite dispersed when mixed with DPPC liposomes (Figure 4D).

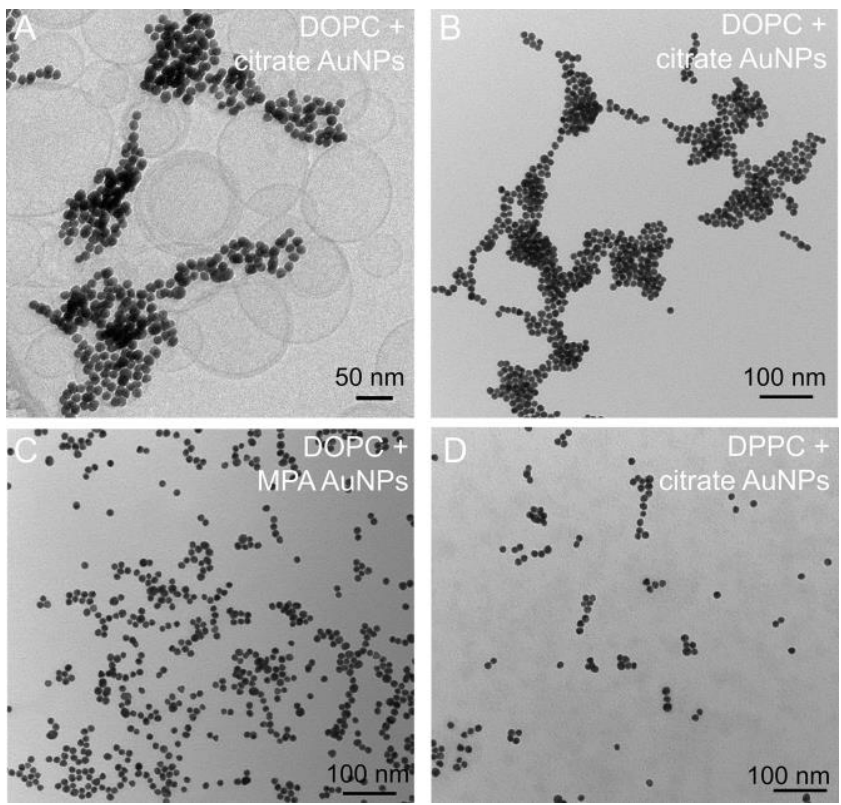

Figure 4. (A) Cryo-TEM and (B) normal TEM micrographs of DOPC liposomes mixed with citrate-capped AuNPs. Normal TEM micrographs of (C) DOPC mixed with MPA-AuNPs and (D) DPPC with citrate-AuNPs.

While lipid fluidity appears to be a good model to explain our observations, new insights can be obtained by quantitative analysis. The diffusion coefficient $\left(D_{\mathrm{L}}\right)$ differs only by a few folds for lipids in different phases. For example, the $D_{\mathrm{L}}$ of DPPC at $45{ }^{\circ} \mathrm{C}$ is $\sim 17.8 \mu \mathrm{m}^{2} \mathrm{~s}^{-1}$, and it is only $\sim 5$-fold smaller at room temperature, where DPPC is in the gel phase. ${ }^{25} \mathrm{~A}$ DPPC molecule can diffuse $4\left(D_{\mathrm{L}} \times t\right)^{1 / 2}=\sim 7.5 \mu \mathrm{m}$ in $1 \mathrm{sec}$ at room temperature. Since the liposome size is only $\sim 100 \mathrm{~nm}$ diameter, this diffusion length scale of individual lipids in $1 \mathrm{sec}$ is much larger than the size of our liposome. Since AuNPs take minutes to change color on DPPC, it is unlikely that individual lipids can carry AuNPs along.

Since citrate-capped AuNPs interact strongly with the lipids, it is reasonable that each AuNP and the underlying lipids form a tight complex (in a sense similar to lipid rafts with decorated AuNPs). Diffusion of this whole complex is slower than that of individual lipids and its diffusion is characterized by a dragging force related to particle size and lipid viscosity. ${ }^{26,27}$ Buffer and temperature that can influence lipid diffusion will also influence the motion of this complex. The viscosity of DPPC is up to $\sim 30$-fold higher than that of DOPC. ${ }^{28}$ While this may better explain the difference ob- served in Figure $2 \mathrm{C}$, it still does not explain why AuNPs have different equilibrium states on different liposomes. For example, for both the MPA-AuNP/DOPC system (Figure 3A) and the citrate-AuNP/DPPC system (Figure $2 \mathrm{C}$ ), the color change stopped after 10-30 min and the AuNPs only formed small clusters (Figure $4 \mathrm{C}, \mathrm{D}$ ). If kinetics (e.g. diffusion and collision frequency) were the only important factor, AuNPs should have reached the same color eventually.

Therefore, we reason that thermodynamic effects may also play a role. Adsorption of citrate-AuNP by PC lipids is so strong that fluid lipids gel at the point of adsorption. The NP adsorption induced lipid gelation was proposed by Granick and co-workers. 9 Adsorption of metal ions can also modulate the phase transition behavior of liposomes. ${ }^{29}$ We believe that citrate-capped AuNPs have the strongest influence due to a very large Hamaker constant and they can be in direct contact with the liposome surface. This is reflected from the elevated $T_{\mathrm{c}}$, and transient liposome leakage upon AuNP adsorption/desorption. ${ }^{21}$ Based on this, we herein propose a more detailed model. When citrate-AuNPs are adsorbed by fluid DOPC, local lipid gelation occurs (Figure $1 \mathrm{D}$, marked by the blue color). The low viscosity of the surrounding DOPC allows faster diffusion of the AuNP/DOPC complex. Aggregation of AuNPs on DOPC decreases the area of fluid/gel membrane interface (merging of the blue lipid region), thus thermodynamically favorably. When AuNPs are adsorbed on the gel phase DPPC, AuNP aggregation is unfavorable both kinetically (i.e. all in gel phase) and thermodynamically (no fluid/gel lipid interface to remove) (Figure $1 \mathrm{E}$ ). For MPAAuNPs on DOPC, the weak adsorption does not induce significant lipid gelation, also limiting their aggregation (Figure ${ }_{1 C}$ ). So both kinetic and thermodynamic effects favor AuNP aggregation on DOPC, but not on DPPC. Normally, citratecapped AuNPs do not aggregate at such low salt concentrations because of charge repulsion. Our model of local gelation and merging of gel phase can also explain surface aggregation of AuNPs on DOPC due to the extra thermodynamic driving force.

\section{Conclusions}

In summary, we reported the favorable kinetic and thermodynamic effects of the fluid DOPC liposomes to promote the aggregation of citrate-AuNPs. Previous studies at the NP/lipid interface mainly focused on the following two scenarios. First, the stage of adsorption (e.g. probed by liposome leakage), ${ }^{21}$ and second, the equilibrium state (e.g. probed by DSC or LAUDEN dye). 9 In this work, we demonstrated the feasibility of probing the new process for liposome/NP conjugates to reach equilibrium. AuNPs are particularly useful for this purpose, and in turn this study allows a new method for probing AuNP surface property. This observation is not only fundamentally interesting, but provides new ways of designing NP self-assembly on fluid lipid surfaces.

\section{ASSOCIATED CONTENT}

\section{Supporting Information}

Materials and methods, DLS and additional displacement and adsorption data. This material is available free of charge via the Internet at http://pubs.acs.org.

\section{AUTHOR INFORMATION}




\section{Corresponding Author}

Email: liujw@uwaterloo.ca

\section{Notes}

The authors declare no competing financial interests.

\section{ACKNOWLEDGMENT}

Funding for this work is from the Natural Sciences and Engineering Research Council of Canada (NSERC).

\section{REFERENCES}

(1) Sackmann, E., Supported Membranes: Scientific and Practical Applications. Science 1996, 271, 43-48.

(2) Yamazaki, V.; Sirenko, O.; Schafer, R. J.; Groves, J. T., Lipid Mobility and Molecular Binding in Fluid Lipid Membranes. J. Am. Chem. Soc. 2005, 127, 2826-2827.

(3) Yoshina-Ishii, C.; Boxer, S. G., Arrays of Mobile Tethered Vesicles on Supported Lipid Bilayers. J. Am. Chem. Soc. 2003, 125, 36963697.

(4) Hartman, K. L.; Kim, S.; Kim, K.; Nam, J.-M., Supported Lipid Bilayers as Dynamic Platforms for Tethered Particles. Nanoscale 2015, 7, 66-76.

(5) Mascalchi, P.; Haanappel, E.; Carayon, K.; Mazeres, S.; Salome, L., Probing the Influence of the Particle in Single Particle Tracking Measurements of Lipid Diffusion. Soft Matter 2o12, 8, 4462-4470.

(6) Deverall, M. A.; Gindl, E.; Sinner, E. K.; Besir, H.; Ruehe, J.; Saxton, M. J.; Naumann, C. A., Membrane Lateral Mobility Obstructed by Polymer-Tethered Lipids Studied at the Single Molecule Level. Biophys. J. 2005, 88, 1875-1886.

(7) Lee, Y. K.; Kim, S.; Oh, J.-W.; Nam, J.-M., Massively Parallel and Highly Quantitative Single-Particle Analysis on Interactions between Nanoparticles on Supported Lipid Bilayer. J. Am. Chem. Soc. 2014, 136, 4081-4088.

(8) Yu, Y.; Anthony, S. M.; Zhang, L. F.; Bae, S. C.; Granick, S. Cationic Nanoparticles Stabilize Zwitterionic Liposomes Better Than Anionic Ones. J. Phys. Chem. C 2007, 111, 8233-8236.

(9) Wang, B.; Zhang, L. F.; Bae, S. C.; Granick, S., NanoparticleInduced Surface Reconstruction of Phospholipid Membranes. Proc. Natl. Acad. Sci. U.S.A. 2008, 105, 18171-18175.

(10) Reimhult, E.; Hook, F.; Kasemo, B., Intact Vesicle Adsorption and Supported Biomembrane Formation from Vesicles in Solution: Influence of Surface Chemistry, Vesicle Size, Temperature, and Osmotic Pressure Langmuir 2003, 19, 1681-1691.

(11) Cho, N. J.; Frank, C. W., Fabrication of a Planar Zwitterionic Lipid Bilayer on Titanium Oxide. Langmuir 2o10, 26, 15706-15710.

(12) Wang, F.; Liu, J., A Stable Lipid/Tioz Interface with Headgroup-Inversed Phosphocholine and a Comparison with $\mathrm{SiO}_{2}$. J. Am. Chem. Soc. 2015, 137, 11736-11742.

(13) Ang, P. K.; Jaiswal, M.; Lim, C. H. Y. X.; Wang, Y.; Sankaran, J.; Li, A.; Lim, C. T.; Wohland, T.; Barbaros, O.; Loh, K. P., A Bioelec-
tronic Platform Using a Graphene-Lipid Bilayer Interface. ACS Nano 2010, 4, 7387-7394.

(14) Ip, A. C. F.; Liu, B.; Huang, P.-J. J.; Liu, J., Oxidation LevelDependent Zwitterionic Liposome Adsorption and Rupture by Graphene-Based Materials and Light-Induced Content Release. Small 2013, 9, 1030-1035.

(15) Wang, F.; Liu, J., Nanodiamond Decorated Liposomes as Highly Biocompatible Delivery Vehicles and a Comparison with Carbon Nanotubes and Graphene Oxide. Nanoscale 2013, 5, 12375-12382.

(16) Van Lehn, R. C.; Atukorale, P. U.; Carney, R. P.; Yang, Y.-S.; Stellacci, F.; Irvine, D. J.; Alexander-Katz, A., Effect of Particle Diameter and Surface Composition on the Spontaneous Fusion of Monolayer-Protected Gold Nanoparticles with Lipid Bilayers. Nano Lett. 2013, 13, 4060-4067.

(17) Hou, W.-C.; Moghadam, B. Y.; Corredor, C.; Westerhoff, P. Posner, J. D., Distribution of Functionalized Gold Nanoparticles between Water and Lipid Bilayers as Model Cell Membranes. Environ. Sci. Technol. 2012, 46, 1869-1876.

(18) Tatur, S.; Maccarini, M.; Barker, R.; Nelson, A.; Fragneto, G., Effect of Functionalized Gold Nanoparticles on Floating Lipid Bilayers. Langmuir 2013, 29, 6606-6614.

(19) Bailey, C. M.; Kamaloo, E.; Waterman, K. L.; Wang, K. F.; Nagarajan, R.; Camesano, T. A., Size Dependence of Gold Nanoparticle interactions with a Supported Lipid Bilayer: A Qcm-D Study. Biophys. Chem. 2015, 203-204, 51-61.

(20) Troiano, J. M.; Olenick, L. L.; Kuech, T. R.; Melby, E. S.; Hu, D; Lohse, S. E.; Mensch, A. C.; Dogangun, M.; Vartanian, A. M.; Torelli, M. D.; Ehimiaghe, E.; Walter, S. R.; Fu, L.; Anderton, C. R.; Zhu, Z.; Wang, H.; Orr, G.; Murphy, C. J.; Hamers, R. J.; Pedersen, J. A.; Gei-
ger, F. M., Direct Probes of 4 Nm Diameter Gold Nanoparticles In- teracting with Supported Lipid Bilayers. J. Phys. Chem. C 2015, 119, 534-546.

(21) Wang, F.; Liu, J., Self-Healable and Reversible Liposome Leakage by Citrate-Capped Gold Nanoparticles: Probing the Initial Adsorption/Desorption Induced Lipid Phase Transition. Nanoscale 2015, 7, 15599-15604.

(22) Šegota, S.; Vojta, D.; Kendziora, D.; Ahmed, I.; Fruk, L.; Baranović, G., Ligand-Dependent Nanoparticle Clustering within Lipid Membranes Induced by Surrounding Medium. J. Phys. Chem. B 2015 $119,5208-5219$.

(23) Hainfeld, J. F.; Powell, R. D., New Frontiers in Gold Labeling. Journal of Histochem. Cytochem. 2000, 48, 471-480.

(24) Bishop, K. J. M.; Wilmer, C. E.; Soh, S.; Grzybowski, B. A., Nanoscale Forces and Their Uses in Self-Assembly. Small 2009, 5, 16001630.

(25) Lindblom, G.; Orädd, G., Lipid Lateral Diffusion and Membrane Heterogeneity. BBA-Biomembranes 2009, 1788, 234-244.

(26) Domanov, Y. A.; Aimon, S.; Toombes, G. E. S.; Renner, M.; Quemeneur, F.; Triller, A.; Turner, M. S.; Bassereau, P., Mobility in Geometrically Confined Membranes. Proc. Natl. Acad. Sci. U.S.A. 2011, 108, $12605-12610$.

(27) Saffman, P. G.; Delbrück, M., Brownian Motion in Biological Membranes. Proc. Natl. Acad. Sci. U.S.A. 1975, 72, 3111-3113.

(28) Nojima, Y.; Iwata, K., Viscosity Heterogeneity inside Lipid Bilayers of Single-Component Phosphatidylcholine Liposomes Observed with Picosecond Time-Resolved Fluorescence Spectroscopy. J. Phys. Chem. B 2014, 118, 8631-8641.

(29) Šegota, S.; Vojta, D.; Pletikapić, G.; Baranović, G. Ionic Strength and Composition Govern the Elasticity of Biological Membranes. A Study of Model Dmpc Bilayers by Force- and Transmission IR Spectroscopy. Chem. Phys. Lipids 2015, 186, 17-29. 
